\title{
The Anchoring Energy of Liquid Crystal Molecules to Magnetic Particles in HAB-Based Ferronematics
}

\author{
N. Tomašovičová, M. Koneracká, P. KopČanský, \\ M. Timko, V. ZÁvišOvÁ \\ Institute of Experimental Physics, Slovak Academy of Sciences \\ Watsonova 47, 04001 Košice, Slovakia
}

A. Vajda, K. Fodor-Csorba, N. Éber, T. Tóth-Katona

Research Institute for Solid State Physics and Optics

Hungarian Academy of Sciences

P.O. Box 49, 1525 Budapest, Hungary

\section{J. JADZYN}

Institute of Molecular Physics, Polish Academy of Sciences

Smoluchowskiego 17, 60-179 Poznań, Poland

In this work we describe the magnetic Freedericksz transition in HABbased $\left(4,4^{\prime}\right.$-di- $n$-heptylazoxybenzene) ferronematics. The ferronematic samples were prepared with two different volume concentrations of magnetic particles $\phi_{1}=2 \times 10^{-5}$ and $\phi_{2}=2 \times 10^{-4}$. The liquid crystal HAB is nematic at the temperatures from $\approx 53^{\circ} \mathrm{C}$ to $70^{\circ} \mathrm{C}$ and smectic $\mathrm{A}$ at the temperatures under $53^{\circ} \mathrm{C}$. The anisotropy of dielectric permitivity of HAB liquid crystal is positive in nematic phase and negative in smectic A phase. The magnetic Fredericksz transition was studied in nematic phase at temperature $60^{\circ} \mathrm{C}$.

PACS numbers: 75.50.Mm, 61.30.Gd, 61.30.Hn

\section{Introduction}

Ferronematics are magnetic colloids based on a liquid crystal matrix and were first suggested on theoretical grounds in 1970 by Brochard and de Gennes [1]. The surface anchoring in the magnetic particles couples the magnetic and nematic order 
and dramatically increases the weak magnetic interaction. The authors predicted a rigid anchoring with $\boldsymbol{m} \| \boldsymbol{n}$, where the unit vector $\boldsymbol{n}$ (director) denotes the preferential direction of the nematic molecules and the unit vector $\boldsymbol{m}$ denotes orientation of magnetic moment of magnetic particles. In the first experiment Rault et al. [2] reported the basic magnetic properties of a suspension of rodlike $\gamma-\mathrm{Fe}_{2} \mathrm{O}_{3}$ particles in $4^{\prime}$-methoxybenzylidene-4- $n$-butylaniline (MBBA) liquid crystal. Later, based on the estimations given by Brochard and de Gennes, first lyotropic [3] and then thermotropic [4] ferronematics were prepared and studied. In the last two decades interest in these materials has grown. Not only because these systems pose interesting physical problems, but also they promise to provide an optical device technology based on magnetic switching [5, 6]. The relevant key properties of a nematic crystal doped with magnetic particles are the intrinsic higher magnetic susceptibility (compared with that of the pure liquid crystal) and the uniform molecular reorientation of the entire liquid crystal matrix, or macroscopic collective behavior, in varying magnetic field. Burylov and Raikher [7] generalized the continuum theory of Brochar and de Gennes to finite nematic anchoring energies on the magnetic particle surface. This explained experiments in which the nematic director $\boldsymbol{n}$ and the magnetic moment of the magnetic particles $\boldsymbol{m}$ were not parallel [4]. Further experiments confirmed the general picture. In this work we describe the magnetic Freedericksz transition in HAB (4,4'-di- $n$ heptylazoxybenzene) based ferronematics.

\section{Experimental}

The samples were prepared by addition of spherical shaped magnetic nanoparticles with a diameter $d=10 \mathrm{~nm}$ into a matrix of thermotropic liquid crystal. The ferronematic samples were prepared with two different volume concentrations of the magnetic particles $\phi_{1}=2 \times 10^{-5}$ and $\phi_{2}=2 \times 10^{-4}$. The calorimetric scans were performed by using a differential scanning calorimetry (DSC) instrument Mettler FP80HT at a scan rate $4^{\circ} \mathrm{C} \mathrm{min}^{-1}$ in the temperature range from $20^{\circ} \mathrm{C}$ up to $90^{\circ} \mathrm{C}$. The liquid crystal $\mathrm{HAB}$ is nematic at temperatures from $\approx 53^{\circ} \mathrm{C}$ to $70^{\circ} \mathrm{C}$ and smectic $\mathrm{A}$ at the temperatures under $53^{\circ} \mathrm{C}$. The anisotropy of the dielectric permittivity of HAB is positive $\left(\varepsilon_{\mathrm{a}}>0\right)$ in the nematic phase and negative $\left(\varepsilon_{\mathrm{a}}<0\right)$ in the smectic A phase. The Freedericksz transition was studied in the nematic phase at $60^{\circ} \mathrm{C}$. The structural transitions in ferronematic samples were detected by capacitance measurements in a capacitor with ITO-coated glass electrodes (LINCAM Co.). The capacitor with the electrode area approximately $1 \mathrm{~cm} \times 1 \mathrm{~cm}$ was placed into a regulated thermostat system, the temperature was stabilized with the accuracy of $0.05^{\circ} \mathrm{C}$. The distance between the electrodes (sample thickness) was $D=5 \mu \mathrm{m}$. The initial texture of the liquid crystal was planar. The capacitance was measured at the frequency of $1 \mathrm{kHz}$ by the high precision capacitance bridge Andeen Hagerling. The applied bias electric field $\boldsymbol{E}_{\mathrm{B}}$ was perpendicular to the surface of the electrodes and an additional external magnetic field was applied perpendicular to the bias electric field. 


\section{Results}

The pure HAB liquid crystal and the HAB-based ferronematics show three transitions according to the DSC curves (Fig. 1). Melting point of pure HAB was found to be $29^{\circ} \mathrm{C}$ where the crystaline phase transforms into the smectic A. The smectic A phase transforms into the nematic phase at $53^{\circ} \mathrm{C}$ and the nematic phase transforms into the isotropic at $70^{\circ} \mathrm{C}$. From Fig. 1 it is seen that the transiton temperatures of the ferronematic samples are shifted to higher values. The calculated surface density of the anchoring energy $W$ at the nematic-
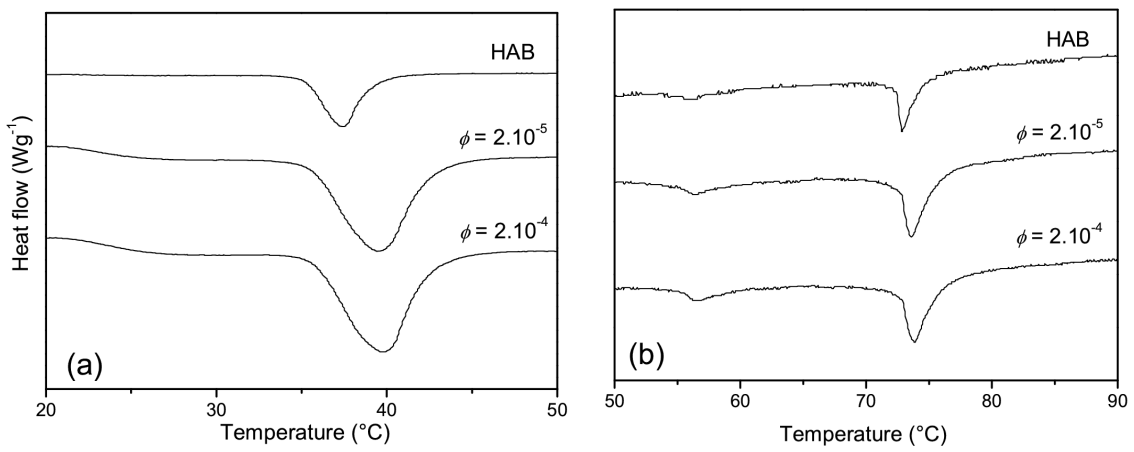

Fig. 1. DSC curves (a) of melting in the pure HAB and the filled systems and (b) of smectic-to-nematic and nematic-to-isotropic transition in the pure HAB and the filled systems. (Both figures belong to the same run but scale on vertical axes is different. The curves are shifted vertically for clarity).

TABLE

The critical magnetic fields $\boldsymbol{B}_{\mathrm{c}}$ measured in pure HAB and in HAB-based ferronematics with volume concentration of magnetic particles $\phi_{1}=$ $2 \times 10^{-5}$ and $\phi_{2}=2 \times 10^{-4}$ at different bias voltage.

\begin{tabular}{c|c|c|c|c|c}
\hline \hline & $U_{\mathrm{B}}[\mathrm{V}]$ & 30 & 40 & 50 & 57 \\
\hline $\mathrm{HAB}$ & $\boldsymbol{B}_{\mathrm{c}}[\mathrm{T}]$ & 5.18 & 7.37 & 9.71 & 12.04 \\
$\phi_{1}$ & $\boldsymbol{B}_{\mathrm{c}}[\mathrm{T}]$ & 4.11 & 4.99 & 6.78 & 10.45 \\
$\phi_{2}$ & $\boldsymbol{B}_{\mathrm{c}}[\mathrm{T}]$ & 2.16 & 2.60 & 3.65 & 8.25
\end{tabular}

magnetic particle boundary is $\approx 10^{-3} \mathrm{~N} \mathrm{~m}^{-1}$ and the parameter $\omega$ defined as a ratio of the anchoring energy to the elastic energy of the liquid crystal is $\approx 10^{-1}$. These results characterize the soft anchoring of nematic molecules on the surfaces of the magnetic particles that permits both types of boundary conditions $(\boldsymbol{m} \| \boldsymbol{n}$ and $\boldsymbol{m} \perp \boldsymbol{n})$. The parallel initial condition $(\boldsymbol{m} \| \boldsymbol{n})$ was supposed. 
The applied bias electric voltage $U_{\mathrm{B}}$ induces a splay Freedericksz transition, rotating the nematic molecules normal to the electrodes. The increasing external magnetic field $\boldsymbol{B}$ reduces this deformation by turning the director back toward the magnetic field. At the treshold magnetic field $\boldsymbol{B}_{\mathrm{c}}$ the initial planar texture is restored, i.e. the electric Freedericksz transition is fully inhibited. In this case the addition of fine magnetic particles should decrease $\boldsymbol{B}_{\mathrm{c}}$. In Table the critical magnetic fields measured at different bias electrical fields are summarized for pure $\mathrm{HAB}$ as well as for HAB-based ferronematic samples with two volume concentrations of the magnetic particles, $\phi_{1}=2 \times 10^{-5}$ and $\phi_{2}=2 \times 10^{-4}$. From this table it is seen that with increasing volume concentration of the magnetic particles the critical magnetic field decreases. This result confirmed the supposed parallel initial boundary condition in HAB-based ferronematics.

\section{Acknowledgments}

This work was supported by the Slovak Academy of Sciences (grant No. 6166), Science and Technology Assistance Agency (APVV) under the contract No. SK-MAD-02606, the Grenoble High Magnetic Field Laboratory, with support of EC Program, by Hungarian Research Funds OTKA K61075, NKFP-128/6, and NKTH/KPf SK-19/2006, and the Slovak-Hungarian bilateral exchange.

\section{References}

[1] F. Brochard, P.G. de Gennes, J. Phys. (Paris) 31, 691 (1970).

[2] J. Rault, P.E. Cladis, J.P. Burger, Phys. Lett. A 32, 199 (1970).

[3] J. Liebert, A. Martinet, J.Phys.Lett. Vol. 40, 363 (1979).

[4] S.H. Chen, N.M. Amer, Phys.Rev.Lett. 51, 2298 (1983).

[5] S.K. Srivatsa, G.S. Ranganath, Phys. Rev. E. 60, 5639 (1999).

[6] C.Y. Matuo, A.M. Figueiredo Neto, Phys. Rev. E. 60, 1815 (1999).

[7] S.V. Burylov, Y.I. Raikher, J. Phys. Lett. A 149, 279 (1990). 